\title{
Fenómeno isquemia/reperfusión en cirugía cardiovascular durante circulación extracorpórea
}

\author{
Ischemia/reperfusion phenomenon in cardiovascular surgery during \\ extracorporeal circulation
}

Fenômeno de isquemia/reperfusão durante a circulação extracorpórea em cirurgia cardiovascular

\author{
Eliana Cerón López $\mathbb{B}^{1}$
}

1 Servicio de Cirugía Cardiovasculary Torácica, Perfusión Cardiovascular, Clínica Guayaquil, GuayaquilEcuador.

\section{Correspondencia a:}

Eliana Cerón López, eceron@clinicaguayaquil.com

Recibido: 11 de septiembre, 2020 Aceptado: 11 de septiembre, 2020 Publicado: 18 de noviembre, 2021

\section{ARTÍCULO DE REVISIÓN}

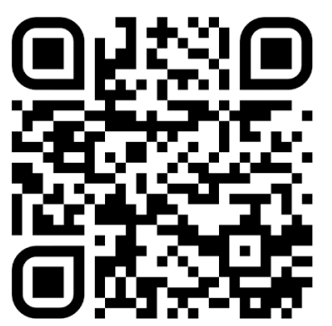

Escanea en tu dispositivo móvil o revisa este artículo en:

https://revistaclinicaguayaquil.org

\section{RESUMEN}

El daño miocárdico por isquemia/reperfusión durante la cirugía cardiaca con circulación extracorpórea, se define como un efecto nocivo, inicialmente debido al pinzamiento aórtico, que genera una isquemia cardiaca, seguido del restablecimiento del flujo sanguíneo a células previamente isquémicas. La cirugía cardiaca ha avanzado durante muchos años con la inclusión de la circulación extracorpórea; sin embargo, su implementación también genera efectos deletéreos en el organismo conllevando a un sinnúmero de reacciones que amplifican el proceso inflamatorio. Debido a que la isquemia y la reperfusión provocan efectos como acidosis metabólica, generación de ácido láctico, arritmias por reperfusión, liberación de radicales libres de oxígeno, entre otros, se ha visto la necesidad de estudiar este fenómeno e intervenir en estrategias, durante la circulación extracorpórea, encaminadas a contrarrestar lo anteriormente mencionado.

Palabras clave: Isquemia; Daño por reperfusión; Circulación extracorpórea; Necrosis por permeabilidad de la transmembrana mitocondrial; Radicales libres

\begin{abstract}
Myocardial damage due to the ischemia-reperfusion injury during cardiac surgery with extracorporeal circulation is defined as a harmful effect; initially due to aortic clamping, generating cardiac ischemia, which is followed by the restoration of blood flow to previously ischemic cells. For many years cardiac surgery has advanced with the inclusion of cardiopulmonary bypass; however, its implementation also generates deleterious effects in the body, leading to reactions that amplify the inflammatory process. Because both, ischemia and reperfusion, cause effects such as metabolic acidosis, generation of lactic acid, arrhythmias, release of oxygen free radicals, it has been necessary to study these phenomena in order to generate strategies to minimize this injury during extracorporeal circulation.
\end{abstract}

Key words: Ischemia; Reperfusion injury; Extracorporeal circulation; Mitochondrial transmembrane permeability-driven necrosis; Free radicals

\section{RESUMO}

O dano miocárdico devido à isquemia/reperfusão durante a cirurgia cardíaca com circulação extracorpórea é definido como um efeito deletério, inicialmente devido ao pinçamento da aorta, levando à 
isquemia cardíaca, seguido pela restauração do fluxo sanguíneo para células anteriormente isquêmicas. A cirurgia cardíaca avançou durante muitos anos com a inclusão da circulação extracorpórea; entretanto, sua implementação também tem efeitos deletérios sobre o corpo, levando a uma série de reações que amplificam o processo inflamatório. Como a isquemia e a reperfusão causam efeitos como acidose metabólica, geração de ácido lático, arritmias de reperfusão, liberação de radicais livres de oxigênio, entre outros, há necessidade de estudar este fenômeno e intervir em estratégias, durante a circulação extracorpórea, com o objetivo de contrariar o acima mencionado.

Palavras-chave: Isquemia; Traumatismo por reperfusão; Circulação extracorpórea; Necrose dirigida por permeabilidade transmembrânica da mitocôndria; Radicais livres

\section{INTRODUCCIÓN}

Los fenómenos de isquemia/reperfusión (I/R) son procesos que alteran el metabolismo de las células cardiacas, secundarios a la isquemia. El cambio de metabolismo aerobio a anaerobio conduce a una ausencia de oxígeno en las células del miocardio, lo que conlleva al daño de las mismas, y además, se suman efectos provocados por la circulación extracorpórea (CEC). Todo esto origina liberación de agentes tóxicos, liberación de radicales libres de oxígeno (RLO) y alteración en los leucocitos polimorfonucleares (PMN). La activación de los PMN induce a mayor daño celular, provocando apoptosis y necrosis de los miocitos, lo que desencadena trastornos en la contractilidad o alteraciones vasculares que pueden tener como resultado un daño severo de órganos, principalmente el corazón y los pulmones, así como también el riñón, cerebro y el intestino (1).

La cirugía cardiaca con CEC ha sido un avance para la especialidad al permitir la corrección de múltiples defectos cardíacos, sin embargo, la perfusión de los demás órganos y el mantener su medio interno en una homeostasis durante estas etapas es un gran desafío para el perfusionista.

Por lo tanto, "la cirugía cardiaca y la CEC en concreto, constituyen un escenario ideal para el estudio del daño por isquemia/reperfusión, al ser procesos reproducibles con una isquemia larga y una reperfusión controlada" (2).

\section{Fenómeno isquemia/reperfusión}

El fenómeno de isquemia/reperfusión en cirugía cardiaca, es un conjunto de sucesos que no se pueden evitar, al ser consecuencia de maniobras indispensables que permiten trabajar dentro del corazón en la mayoría de las intervenciones quirúrgicas. La alteración metabólica es el evento inicial de todo el proceso que desencadena un daño endotelial; este proceso se inicia con el ingreso a CEC, continúa con la posterior isquemia, y culmina con la reperfusión celular.

Durante la cirugía cardiaca con CEC, el corazón se aísla de la circulación, esto inevitablemente induce a isquemia miocárdica. Además de este insulto isquémico, se producirá un golpe adicional después de la reperfusión, lo que puede empeorar la extensión del daño tisular y la disfunción orgánica. A lo largo de los años, se han desarrollado varias estrategias que tienen como objetivo atenuar y/o modular el alcance de esta lesión por I/R relacionada con la CEC (3).

Hoy en día se ha demostrado que estos procesos de I/R se asocian a la apoptosis y necrosis de la célula cardiaca, y es más común cuando la isquemia es seguida de un periodo de reperfusión, que cuando se trata de una isquemia aislada (4).

\section{Isquemia cardiaca}

Partiendo de que el metabolismo cardiaco es netamente aerobio, con un consumo de oxígeno entre $60-150 \mathrm{mmol} / \mathrm{min}$ en seres humanos, y teniendo en cuenta que el principal sustrato energético para la producción de Adenosín Trifosfato (ATP) en el corazón son los ácidos grasos, generando 146 moléculas de ATP a partir de la fosforilación oxidativa de estos, se puede deducir que el requerimiento energético para la función cardiaca es bastante elevado (5).

El ciclo de Krebs y la B-oxidación de ácidos grasos generan moléculas altamente energéticas (nicotinamida adenina dinucleótida - flavín adenín dinucleótido, NADH-FADH) las cuales se oxidan a NAD+-FAD+ cediendo electrones a complejos proteicos (NADH deshidrogenasa I, succinato deshidrogenasa II, Citocromo bc-1III, Citocromo oxidasa IV) y al gran complejo del ATP sintasa (ubiquinona Q10, Citocromo C) que se encuentra en las crestas de la membrana interna de la mitocondria; seguido de un bombeo de protones al espacio intermembranoso, quedando éste en estado de acidosis (5). Los electrones vuelven a la matriz mitocondrial uniéndose a media molécula de oxígeno, proveniente de la ventilación pulmonar, y a dos protones, generando agua. Además, los protones liberados al espacio intermembranoso necesitan ser devueltos a la matriz mitocondrial 
a través del complejo ATP sintasa, el cual fosforila ADP creando ATP, proceso que es conocido como fosforilación oxidativa (5).

Durante la isquemia cardiaca, al no haber cantidad suficiente de oxígeno o inadecuado aporte de oxígeno al corazón debido a la reducción en el flujo sanguíneo, y con ello aporte deficiente de energía para cubrir las necesidades metabólicas y la demanda de oxígeno, los electrones provenientes de las moléculas energéticas son entregados a otras moléculas que generan especies reactivas de oxígeno (ERO) y radicales libres de oxígeno (RLO), ocasionando daño tisular (2).

Además, en esta fase de isquemia, el metabolismo, que en condiciones normales era aerobio, se convierte en metabolismo anaerobio; con esto, la fosforilación oxidativa y la cadena respiratoria mitocondrial se ven afectadas, de igual manera se disminuye la oxidación del piruvato y de los ácidos grasos (2).

El cardiomiocito, al tener la necesidad de obtener energía, lo realiza mediante la glucólisis anaerobia; esta fuente no es muy efectiva en la producción de energía, ya que no satisface las necesidades de la célula al producir solamente de 2 a 3 moléculas de ATP, inclusive en la isquemia provocada por el pinzamiento aórtico en cirugía cardiaca con CEC. Así también, al administrar la cardioplejia fría tampoco se logra un aumento de ATP, ya que el paro hipotérmico genera depleción de los fosfatos de alta energía y altera la función contráctil del miocardio. Esta declinación en la producción y concentración de ATP es lo que lleva a una cascada de fenómenos o reacciones como: aumento del ADP, AMP, adenosina, inosina, hipoxantina, xantina y urato; producción de lactato y acidosis; disminución de la Beta-oxidación de los ácidos grasos libres por la inhibición de la enzima transferasa de acilcarnitina, que es necesaria en el transporte de Acil-Coa desde el citosol a la mitocondria; y alteración de la bomba sodio/potasio, produciéndose un aumento de la concentración celular de sodio y por consiguiente de calcio, a causa del intercambio sodio/calcio, esto se debe al intento de la célula por restablecer su $\mathrm{pH}$ normal. Por consiguiente, el exceso de calcio intracelular, interfiere en la capacidad de las mitocondrias de generar ATP y en la activación del fenómeno de transición de permeabilidad mitocondrial (TPm) (2-6).

Haworth y Hunter describen este fenómeno de TPm como un cambio abrupto en la permeabilidad de la membrana mitocondrial interna, como consecuencia a la concentración de calcio, estrés oxidativo y depleción de ATP. Esta TPm sucede gracias a la apertura de un poro llamado poro de transición de permeabilidad mitocondrial (PTPm), que se encuentra entre la membrana interna y externa que permite el paso libre de moléculas de $<1,5 \mathrm{kD}$ incluyendo protones. Además, según diferentes estudios, se ha demostrado que dicho poro es sensible al aumento de calcio, al pH, a cambios de voltaje de la membrana y a la depleción de nucleótidos de adenina, respondiendo a la inhibición del poro ante la acidosis y depresión de ácidos nucleótidos, y su activación ante la concentración de calcio y RLO $(4,6,7)$. La apertura del PTPm es regulado por la familia de proteínas $\mathrm{Bcl}-2$ que se sintetizan en la membrana mitocondrial y juegan un rol importante tanto pro o anti apoptótica, que permiten aumentar o disminuir la permeabilidad del PTPm permitiendo la vida o la muerte celular (5-8). Una de las consecuencias de la apertura de este poro, es que toda molécula de bajo peso de tipo osmolítico pasa a través de la membrana interna, y así, retiene más proteínas en la matriz mitocondrial que en el citosol. Estas proteínas ejercen un poder coloidosmótico, lo que provoca edema de la matriz mitocondrial, sin embargo, esta estructura se acomoda al incremento de volumen sin romperse; por el contrario, la membrana externa, al tener menos superficie, no tolera el aumento de volumen por lo que se ocasiona una ruptura de la misma (4).

Las proteínas sintetizadas Bax-Bak, de acción proapoptótica, que facilitan la liberación de citocromo $C$ hacia el citosol, al trasladarse al núcleo favorecen la formación de moléculas provocando activación de caspasas, involucradas en la activación de citoquinas proinflamatorias. Sin embargo, las proteínas antiapoptóticas Bcl2 y Bcl-XL evitan este proceso, inhibiendo la formación del PTPm al intervenir en la asociación de proteínas proapoptóticas con el PTPm, evitando la liberación de citocromo $\mathrm{C}$ y por ende el daño celular (5-8).

Existen múltiples causas de isquemia durante la cirugía cardiaca con CEC, una de las causas principales es el pinzamiento aórtico; y el poder visualizar estrategias que prevengan $y / 0$ minimicen el daño y proveer protección durante esta etapa es muy importante. Estos objetivos van encaminados a minimizar la utilización de fosfatos de alta energía y la acumulación de calcio intracelular que es ocasionado por el pinzamiento. Dentro de las intervenciones que 
se plantean están, la hipotermia, administración de cardioplejia, bloqueo de los canales de calcio y reducir el tiempo de isquemia, el cual depende del tipo de procedimiento, la habilidad del cirujano y las posibles complicaciones que se puedan presentar (2).

\section{Reperfusión}

El daño miocárdico por reperfusión es definido por algunos autores como el efecto adverso producido al restablecerse la circulación y propiciar la llegada de sangre, oxígeno y nutrientes a las células previamente isquémicas. La restauración del flujo sanguíneo puede acompañarse de RLO, acumulación de calcio intracelular y alteración en el metabolismo celular; estas situaciones pueden condicionar un daño miocárdico funcional y estructural. Clínicamente este daño puede manifestarse después del restablecimiento de la circulación coronaria previamente bloqueada, o después del uso de la bomba extracorpórea en cirugía cardíaca, manifestándose como miocardio aturdido o hibernado (9).

Nieves define el daño por reperfusión como la incapacidad del corazón para utilizar normalmente el oxígeno, debido al suministro reducido en la mitocondria causado, ya sea por el deterioro funcional del metabolismo oxidativo o por daño estructural (6). La reperfusión está asociada con arritmias, formación de RLO y flujo anormal de calcio (6).

Por lo tanto, en la $I / R$, y especialmente en la fase de reperfusión, los complejos I y III de la cadena respiratoria de transporte de electrones mitocondrial son los responsables de producir RLO (10). Los RLO son moléculas eléctricamente inestables, debido a que en su última capa ha desaparecido un electrón o más y se destacan tanto por su abundancia como por su lesividad. Entre los RLO están: el radical superóxido (O2-) que, a pesar de ser una especie reactiva primaria es altamente tóxico al interactuar con otras moléculas y generar ERO secundarias; el peróxido de hidrógeno que se forma por dismutación del anión superóxido, reacción catalizada por el superóxido dismutasa o directamente por reducción bivalente del oxígeno; y el radical hidroxilo que es una de las especies más reactivas y se forma a partir de la descomposición del peróxido de hidrógeno en presencia de metales como hierro y cobre. De igual manera se forman radicales libres de nitrógeno como el óxido nítrico $(\mathrm{ON})$, peroxinitritos y dióxido de nitrógeno; además, se ha encontrado otras vías generadoras de ERO en el miocardio isquémico, entre ellas el $\mathrm{NADPH}$ oxidasa y la xantina oxidasa, presente en la membrana plasmática de las células fagocíticas (10).

Debido a que los RLO no son intrínsecamente deletéreos; de hecho, nuestro propio cuerpo los produce en cantidades moderadas para luchar contra bacterias y virus, el efecto nocivo aparece cuando se exceden en el número y ocurre la infiltración de neutrófilos durante la reperfusión (11).

A pesar del papel fisiológico que desempeñan algunos RLO, también pueden producir reacciones de oxidación indeseadas como: desnaturalización de cadenas de DNA, liberación de factores quimiotácticos que provocan la llegada de leucocitos activados, que a su vez generan más radicales libres; inicio de la peroxidación lipídica, que genera hidroperóxidos, aldehídos y endoperóxidos, con daño en las proteínas y DNA; y desnaturalización de las proteínas presentes en el citosol y las enzimas de membrana, incluyendo un inhibidor de alfa-1-antitripsina (2).

Una vez superado el periodo de isquemia, y al restaurarse el flujo al corazón, existe una rápida elevación del calcio mitocondrial; la reentrada de calcio al interior de una célula miocárdica, produce un daño celular severo caracterizado por una marcada elevación de las enzimas cardíacas y la formación de las denominadas bandas de contracción. Se ha logrado identificar que las concentraciones elevadas de calcio en el interior de la célula conducen a la necrosis de ésta, y además una relación con la sobreexpresión del gen del receptor beta adrenérgico (9).

Entre los eventos posteriores a la reperfusión, las células al ser privadas de oxígeno, se produce una disminución de los valores celulares de ATP y un incremento del fosfato, alteración local del sistema de renina-angiotensina cardíaca, lo que limita la salida del calcio de la célula conllevando a la acumulación de éste catión en su interior, incrementando así la permeabilidad de la membrana y la apertura rápida del PTPm, perdiendo la capacidad para restablecer la homeostasis del calcio y finalmente provocar daño celular con posterior disfunción ventricular (9).

Crompton y colaboradores fueron los primeros en demostrar que la apertura del PTPm juega un papel crucial en la I/R miocárdica. Aunque en 
general, se acepta que la apertura del PTPm se produce en la reperfusión, pero algunos estudios sugieren que también durante la isquemia (12). Durante la reperfusión se ha considerado que la inhibición del PTPm representa un mecanismo poderoso, a través del cual el corazón puede ser protegido contra la injuria por I/R, por lo tanto el PTPm es un punto crucial para el diseño de estrategias terapéuticas cardioprotectoras (6).

Por otra parte, la alteración estructural, evidencia alteración en el funcionamiento, manifestándose clínicamente en un principio con arritmias, generalmente ventriculares, que se ven influenciadas por diferentes mecanismos, entre estos la duración de la isquemia, oscilaciones de las concentraciones de calcio citosólico, flujo de catecolaminas por la isquemia e incluso el flujo de la reperfusión junto con la liberación de RLO (13).

Lo anterior podría desencadenar una disfunción ventricular, que en principio podría manifestarse como un miocardio aturdido caracterizado por un flujo coronario ya restablecido y una función contráctil reversible con el tiempo; de lo contrario, cuando hay un flujo sanguíneo deteriorado junto con la disfunción de la contractilidad se está hablando de un miocardio hibernante. Si la reperfusión ocurre antes de que el daño sea irreversible, la célula puede recuperarse. Sin embargo, en contra de lo esperado, algunas células miocárdicas sufren mayor daño una vez que el flujo coronario se ha restablecido (9).

\section{Estrategias cardioprotectoras para atenuar el daño por reperfusión}

Estas causas presentes durante los dos fenómenos, especialmente el estrés oxidativo junto con la liberación de RLO, pueden ser controladas por un sistema antioxidativo; este sistema consta de una serie de sustancias antioxidantes que juegan un papel importante al ser capaces de neutralizar la acción oxidante de los RLO mediante la liberación de electrones en nuestra sangre, los que son captados por los mismos (11).

El sistema antioxidante primario está compuesto fundamentalmente por las enzimas como la catalasa, superóxido dismutasa (SOD) y las enzimas del ciclo redox del glutatión (2). Es así que, los autores de un estudio realizado a pacientes pediátricos afirman que los corazones de pacientes con patologías cianosantes se asocian con antioxidantes endógenos agotados (glutatión peroxidasa, SOD y catalasa), y por lo tanto, son más susceptibles a la lesión miocárdica por I/R durante la cirugía a corazón abierto, en comparación a pacientes de patologías no cianóticas (14).

Como ya se mencionó anteriormente, en la fase de reperfusión es donde hay más liberación de RLO, en contraposición a esto, existen agentes barredores o scavengers, que son especies químicas cuya posibilidad antioxidante reside en su capacidad para destruir directamente los RLO (15). También existen enzimas que inhiben o disminuyen los radicales citotóxicos; se ha documentado que estos scavengers son más efectivos si se administran como un pretratamiento o adicionados a las soluciones cardiopléjicas (16).

Se han utilizado distintas sustancias de tipo radical scavengers, entre ellas están el alopurinol y oxipurinol, para inhibir la xantina-oxidasa y de esta manera mejorar la función post-isquemia; otros agentes utilizados en forma experimental son catalasa, glutatión reducido, deferoxamina, manitol, y glutatión peroxidasa (16).

En un estudio prospectivo, 45 pacientes fueron evaluados para determinar el efecto de la CEC en la generación de RLO. Los pacientes fueron separados en 3 grupos, 20 de ellos fueron grupo control (grupo I), a los siguientes 15 pacientes se administraron antioxidantes exógenos de RLO (manitol) antes de entrar en CEC (grupo II), y al resto de pacientes se administró alopurinol (grupo III) (17). El resultado de este estudio concluyó en que el grupo I, los niveles plasmáticos de peróxido de hidrógeno ( $\left.\mathrm{H}_{2} \mathrm{O} 2\right)$ aumentó durante la CEC de $65 \pm 6$ a $125 \pm$ $12 \mathrm{microM} / \mathrm{ml}(p<0.001)$, no siendo así en el grupo II, donde fueron significativamente más bajos ( $p<0.03)$, y en el grupo III, niveles aún más bajos en comparación con los del grupo I $(p<0.05)$. Los niveles de $\mathrm{H} 2 \mathrm{O} 2$ en los glóbulos blancos disminuyeron en el grupo I $(p<0.04)$ y en el grupo II durante la CEC, mientras que no se obtuvieron concentraciones intracelulares de $\mathrm{H} 2 \mathrm{O} 2$ en pacientes del grupo III. Se llegó a la conclusión que los RLO generados durante la CEC se pueden minimizar mediante el pretratamiento, con antioxidantes como el manitol y alopurinol, evitando la peroxidación lipídica de biomembrana (17).

De igual manera, el objetivo de la protección miocárdica es también minimizar el daño por la reperfusión; por lo que algunos autores afirman que la cardioplejia sanguínea contiene numerosos antioxidantes, al mismo tiempo 
transporta oxígeno a través de la hemoglobina en los glóbulos rojos. Además, los glóbulos rojos contienen actividad enzimática para remover RLO, que han demostrado ser importantes. Entre otras cosas, las proteínas sanguíneas tienen capacidad amortiguadora o buffer, especialmente sus grupos histidina e imidazol, así como también contiene mejores propiedades oncóticas que podrían disminuir el edema, además de contener sustratos metabólicos (18).

Otros autores afirman que la administración de cardioplejia caliente restablece una serie de funciones como: reabastece al miocardio de sustratos metabólicos con anterioridad al inicio de la función mecánica, ayuda a mejorar la recuperación metabólica, y permite lavar productos de la isquemia (13).

Además, la presión de reperfusión tras el despinzamiento, es importante para limitar el daño miocárdico. Durante el primer y segundo minuto no se debe producir un aumento brusco de la presión, y después de esos dos minutos se debe procurar evitar la hipertensión, debido a que el endotelio se lesiona durante la isquemia y sus propiedades vaso reguladoras se ven limitadas, empeorando si la presión aumenta. Así también, se debe evitar la distensión ventricular, manteniendo descomprimido el ventrículo, así como desfibrilación precoz, para atenuar el agotamiento fulminante de los sustratos. La correcta extracción del aire intracardiaco para evitar la embolia aérea en arterias coronarias, vasos cerebrales o periféricos, especialmente la coronaria derecha, que es más vulnerable debido a su localización anterior en la raíz aórtica. Durante los cinco y diez minutos después de la reperfusión, una relativa hipocalcemia ayuda a prevenir la entrada masiva y súbita de calcio a las células miocárdicas, después de este periodo, la membrana celular es restaurada, por lo que es segura la administración del mismo (13).

Por otro lado, se ha descrito que al inhibir el PTPm, en el contexto de $I / R$, tiene un efecto protector en el miocardio. Investigaciones han demostrado que el administrar medicamentos que demuestren la capacidad de inhibir la apertura del PTPm y que además resulten seguros para el uso clínico, es muy beneficioso para el corazón; entre estos medicamentos están los anestésicos inhalatorios de tipo halogenado como el sevoflurano, que ha demostrado proteger al corazón, y el pos acondicionamiento con este fármaco, demuestra que tiene un efecto protector contra el daño por I/R, inhibiendo la apertura del poro, aunque los mecanismos a los que se debe estos resultados aún son inciertos (4).

En un estudio realizado en Lituania en el año 2015 se evaluaron las propiedades protectoras del sevoflurano y propofol sobre la actividad de la función mitocondrial y el estado energético del corazón humano relacionada con la lesión por I/R. Para ello evaluaron los biomarcadores de daño miocárdico haciendo un análisis de troponina I antes de la inducción de la anestesia y 12 horas después de la cirugía. En cuanto a la función mitocondrial realizaron un experimento con fibras de tejido auricular derecho humano de 6-10 mg de biopsias tomadas antes de la cardioplejía y a los 10-15 min de reperfusión añadiendo soluciones con sustratos e inhibidores para evaluar la función mitocondrial y la integridad de la membrana externa mitocondrial agregando el efecto del citocromo c exógeno. Se tomó una muestra de 72 pacientes sometidos a cirugía coronaria, los cuales se dividieron en 2 grupos, en el primer grupo (S) se administró de 2-3\% de volumen de sevoflurano y al segundo grupo (P) se administró $2-3 \mathrm{mg} / \mathrm{kg}$ de propofol en la anestesia durante la cirugía (19). La conclusión del estudio demostró que todos los pacientes presentaron liberación anormal de troponina I a las 12 horas del postoperatorio con diferencias estadísticas entre los grupos. La concentración de Troponina del marcador de daño miocárdico fue de $2.2 \pm 0.8 \mathrm{ng} / \mathrm{ml}$ en el Grupo S y de $3.5 \pm 11$ $\mathrm{ng} / \mathrm{ml}$ en el Grupo P ( $<<0,001)$. Además, se demostró que durante el periodo de $\mathrm{I} / \mathrm{R}$ se lesionó la membrana externa mitocondrial y se produjo pérdida del Citocromo C, esta membrana externa mitocondrial fue menos afectada en grupo tratado con sevoflurano en comparación con el grupo de propofol (19).

Según C. Nieves "sería beneficioso mantener un ambiente acidótico al menos al inicio de la reperfusión" (6), esto con el fin de prevenir la apertura del PTPm, evitando las consecuencias que esto conlleva y con la finalidad de mejorar la contractilidad ventricular manteniendo una buena hemodinamia.

\section{Circulación extracorpórea}

A todo este proceso de I/R se suma la lesión provocada por la CEC, ya que la sangre cuando toma contacto con superficies extrañas, libera una serie de sustancias debido a la activación del sistema de complemento. Hace más de 20 años, Chenoweth y su grupo reportó la activación del sistema de complemento durante la CEC en 
adultos, y dos años más tarde, Kirklin demostró la relación entre la activación del complemento y la morbilidad postoperatoria en adultos y en niños, permitiendo una amplificación de la respuesta humoral al generar toxinas anafilácticas como C3 y C5 que al convertirse en complejo C5b-9 generan la liberación de histamina y sustancias vasodilatadoras, que incrementan la permeabilidad vascular del lecho pulmonar (20).

El uso de CEC se asocia con la inducción de una respuesta inflamatoria sistémica, que puede resultar en daños y disfunciones, no sólo del corazón, sino también de otros órganos, uno de ellos es el pulmón. El daño pulmonar agudo ha sido asociado con una mortalidad del $50 \%$. Existen evidencias histológicas que demuestran que la CEC causa profundo daño intersticial, endotelial y epitelial. Más del $50 \%$ de los neutrófilos circulando, son secuestrados en los capilares pulmonares durante la isquemia y el recalentamiento con la subsecuente degranulación, lo que contribuye al daño endotelial vascular de los pulmones (21).

Por otra parte, este órgano que en cirugía cardiovascular está aislado, obliga a estar bajo una ventilación mecánica y a fuerzas inusuales, que son particularmente relevantes para el daño pulmonar y que incluyen la sobredistensión o colapso repetitivo sobre las estructuras bronquiales terminales y de los alvéolos, flujos turbulentos de gases o fluidos sobre la mucosa bronquial (22).

Durante la cirugía cardiaca, la I/R del pulmón posterior a CEC y la activación del complemento, neutrófilos y citoquinas proinflamatorias, imponen una seria agresión pulmonar adicional que puede terminar por comprometer seriamente la dinámica ventilatoria y el intercambio gaseoso (Figura 1) (22).

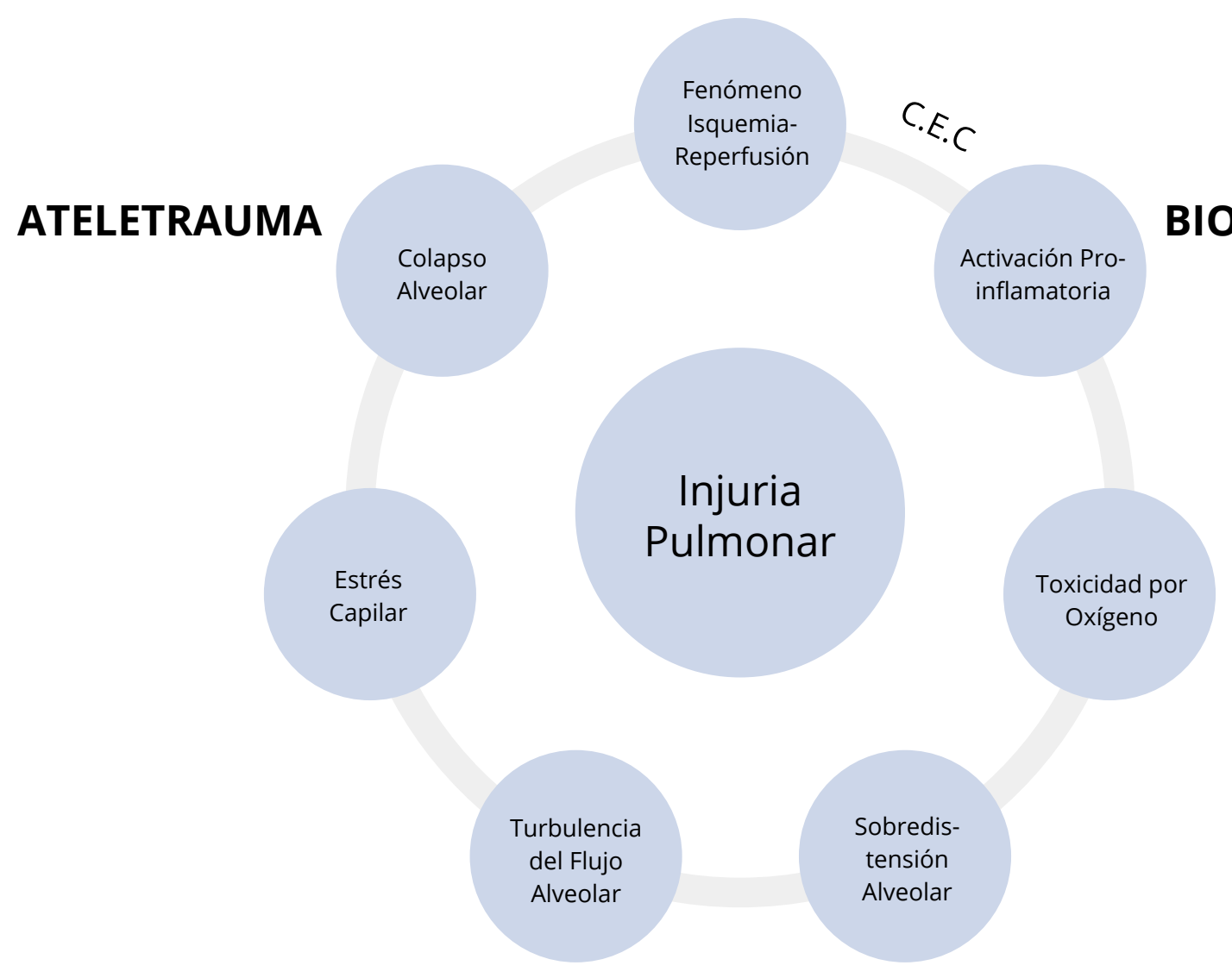

BARO/VOLUTRAUMA

Figura 1. Mecanismos implicados en la generación de injuria pulmonar durante cirugía cardiaca con CEC. 
De igual manera, estas sustancias liberadas por la activación del sistema de complemento actúan sobre los PMN, la interacción entre el neutrófilo y el endotelio involucra moléculas de adhesión (selectinas, B2 integrinas, e inmunoglobulinas); estas moléculas forman coordinadamente una secuencia de eventos denominados, rodamiento, adhesión y migración transendotelial del neutrófilo ya activado, liberando productos citolíticos como leucotrieno, factor activador plaquetario, mieloperoxidasa, colagenasa, elastasa, ácido hipocloroso, anión superóxido (23). Estas endotoxinas también activan a los monocitos, que al unirse al tejido lesionado se convierten en macrófagos; es decir monocitos activados, que a su vez causan producción de citoquinas proinflamatorias como TNF, IL-1, IL6 , IL-8. Es de resaltar que niveles elevados de IL-6 se relacionan con el daño de disfunción miocárdica y con la isquemia provocada por el pinzamiento aórtico (23).

Hennein y colaboradores, mediante un puntaje de motilidad del músculo cardiaco evaluada mediante ecocardiograma transesofágico, concluyeron que aquellos pacientes que no mejoraban la motilidad cardíaca, y por ende la función ventricular, tuvieron niveles de IL-6 10 veces más elevados que aquellos que mejoraron el movimiento regional (24).

Por otra parte, otro órgano que puede verse afectado es el riñón que, en condiciones fisiológicas, recibe aproximadamente el 20\% del gasto cardiaco, aporte que es adecuado para mantener su función. Durante periodos de isquemia es incapaz de sobrevivir sin algún grado de lesión debido a que la tensión dentro de la médula es baja y cualquier alteración en el flujo sanguíneo relacionada con disminución de la presión arterial o del hematocrito puede afectarlo. De igual manera, la corteza renal es muy sensible a la injuria durante la fase de recalentamiento del procedimiento quirúrgico, debido a la presencia de hipoperfusión, durante este periodo el descenso de la presión arterial puede llevar a la reducción de la tasa de filtración glomerular $(6,21)$.

Además, la respuesta inflamatoria sistémica, como resultado del contacto directo de las células sanguíneas con la superficie artificial del circuito extracorpóreo, altera el flujo sanguíneo regional y el tono vasomotor de los riñones, contribuyendo a la lesión renal. La hemólisis durante CEC es un mecanismo nefrotóxico bien reconocido que se produce por destrucción mecánica de eritrocitos debido al contacto con la superficie del circuito extracorpóreo, hiperflujo, condiciones de presión, prolongación del tiempo de CEC, y la aspiración excesiva durante la cirugía, pues la interfase aire sangre producida por la succión, incrementa la fragilidad capilar $(6,21)$.

\section{Estrategias para atenuar el daño por la CEC}

Por lo tanto, la cirugía cardiaca y la perfusión, actualmente han logrado avanzar tanto en las técnicas quirúrgicas, convirtiéndolas en procedimientos mínimamente invasivos, y se han propuesto varias estrategias que tienen como objetivo atenuar los efectos deletéreos de la respuesta inflamatoria. Estos incluyen intervenciones farmacológicas, mejor diseño de los componentes del circuito extracorpóreo, uso de filtros leucocitarios, uso de circuitos de bypass cardiopulmonar miniaturizados, circuitos de hemofiltración, técnicas de ahorro sanguíneo y de hemoadsorción (23).

En la actualidad existen diversos sistemas de oxigenadores que están diseñados para reducir la hemodilución, minimizar la superficie de contacto con la sangre, evitando al máximo los micro émbolos gaseosos utilizando filtros arteriales, disminuir el uso de vasopresores debido a la mejora en la biocompatibilidad, disminuir el traumatismo sanguíneo con recubrimientos fisiológicos como: Carmeda, Trillium, fosforilcolina, Bioline, lo que ayuda a reducir la activación de sustancias proinflamatorias, adsorción de proteínas y adherencia de células, ya descritas anteriormente (25).

\section{Hemofiltración}

Los pacientes sometidos a CEC, desarrollan frecuentemente una sobrecarga hídrica, así como alteraciones electrolíticas, además, el contacto con superficies no endoteliales y el propio trauma quirúrgico promueven el aumento de la permeabilidad capilar con el consecuente desplazamiento de agua al tercer espacio (24).

La hemofiltración consiste en el movimiento del agua a través de una membrana semipermeable como resultado de un gradiente de presión hidrostático o presión transmembrana. A medida que se difunde el agua, se crea un gradiente de concentración de solutos entre la sangre y el otro lado de la membrana. Los solutos siguen el gradiente de concentración siendo transferidos de la sangre al ultrafiltrado, en un proceso de 
extracción de solutos Ilamado convección. El líquido eliminado durante la ultrafiltración se denomina ultrafiltrado o agua plasmática (24).

La introducción de un sistema de ultrafiltración en la CEC, es una alternativa accesible tanto en cirugía cardiaca pediátrica como en adulto, con el fin de combatir la respuesta inflamatoria y disminuir los daños por I/R, así como también disminuir la sobrecarga de líquidos, eliminar mediadores de respuesta inflamatoria, disminuir la necesidad de transfusiones sanguíneas, revirtiendo los efectos de la hemodilución, mejorando así el estado hemodinámico del paciente (13).

Un hemofiltro, es un dispositivo que está constituido por una fibra hueca de polisulfona altamente permeable, actualmente son filtros con capilares de 5.000 fibras y una superficie que va desde 0.25 a $1 \mathrm{~m} 2$; la alta permeabilidad permite el flujo de agua y de solutos con un peso molecular inferior a $50.000 \mathrm{D}$, por lo que no permite la eliminación de proteínas como la albúmina (25).

Propuestas más recientes han dado un gran valor a la hemofiltración modificada, introducida por Naik, Knight y Elliot, al ser una técnica que mejora la estabilidad hemodinámica y la oxigenación temprana postoperatoria, reduce el sangrado y la duración de ventilación mecánica en cirugía pediátrica, además de disminuir la hipertensión pulmonar después de cirugía cardiaca en congénitos, posiblemente al facilitar la extracción de endotelina-1 $(13,21)$.

La hemofiltración modificada al realizarse después de CEC, mejora la fracción de eyección del ventrículo izquierdo y la distensibilidad diastólica, aumenta la presión arterial, disminuye el requerimiento de inotrópicos y reduce el edema en el postoperatorio en los niños (2).

\section{Técnicas de ahorro sanguíneo}

Hablar de cirugía cardiaca y de CEC es también hablar de bancos de sangre. La sangre es uno de los elementos indispensables para la intervención quirúrgica, es uno de los principales componentes afectados debido a la alteración que se produce en la coagulación, al estar expuesta ante superficies no endoteliales, y a ello se le suma la necesidad de hemodilución causada por el mismo circuito extracorpóreo.

Aunque la anemia dilucional severa puede inducir a lesión isquémica de los órganos, las transfusiones de concentrados de glóbulos rojos tienen sus riesgos y puede estar asociada con una mayor morbilidad y mortalidad, por lo que colocar en una balanza riesgo beneficio es un reto para el perfusionista y el equipo de trabajo (26).

A estos riesgos que ya son conocidos y han sido estudiados continuamente por sus efectos deletéreos y su repercusión en el postoperatorio, se le suma situaciones como las creencias religiosas, insuficiente disponibilidad de hemoderivados, pacientes con niveles de hemoglobina bajos no acondicionados previo a cirugía, y pacientes de bajo peso, han llevado a tener la necesidad de buscar estrategias que permitan minimizar el requerimiento de hemocomponentes. Para ello, en el año 2007 las Sociedades de Cirujanos Cardiotorácicos, Anestesiólogos Cardiovascularesy Perfusionistas de diferentes centros, encabezados por el Víctor Ferraris, presidente de la Sociedad de Cirujanos Torácicos, preocupados por el impacto de la transfusión en cirugía cardiaca, conformaron un grupo de trabajo y crearon las Guías de Práctica Clínica sobre la Conservación Sanguínea en Cirugía Cardiaca y después de tres años las actualizaron donde describieron las distintas actuaciones para el ahorro de sangre en pacientes sometidos a cirugía cardiaca (26).

\section{Intervenciones preoperatorio: valoración del paciente}

Se dice que la trombosis es la causa de muerte y la responsable de los infartos y accidentes cerebrovasculares, esporestoquemedicamentos como antiplaquetarios y anticoagulantes juegan un papel muy importante para su tratamiento incluyendo la cirugía cardiaca (27).

La trombosis arterial, responsable del infarto agudo de miocardio (IAM) e ictus isquémico, caracterizada por la presencia de coágulos ricos en plaquetas y con escasa fibrina denominados (trombos blancos), que se generan en lugares de lesión vascular y se previenen y tratan con agentes antiplaquetarios. Sin embargo, como la trombina, principal enzima del sistema de coagulación, es un potente activador plaquetario, también se emplean anticoagulantes para prevenir la trombosis arterial. La enfermedad tromboembólica venosa, que incluye la trombosis venosa profunda y la embolia pulmonar, constituye la tercera causa de mortalidad cardiovascular tras el IAM. Los trombos venosos resultan de una activación de la coagulación y están constituidos principalmente por fibrina, en la que quedan atrapados los glóbulos rojos, 
y escasas plaquetas denominados (trombos rojos); se previenen y tratan generalmente con agentes anticoagulantes (27).

Fármacos antiplaquetarios como la aspirina y el clopidogrel se han consolidado como tratamiento estándar para el síndrome coronario agudo, la aspirina al inhibir la ciclooxigenasa y el clopidogrel al ser antagonistas selectivos del receptor P2Y12 del ADP. Dentro de los nuevos inhibidores de P2Y12 del ADP está el ticagrelor que actúa de forma reversible y no requiere metabolización hepática, siendo un potente inhibidor de la agregación plaquetaria. El cilostazol, al ser un medicamento inhibidor de la fosfodiesterasa III, actúa como antitrombótico y tiene un efecto como dilatador vascular. Se sugiere la suspensión de los fármacos de acuerdo a su tiempo de aclaramiento; la aspirina debe suspenderse entre 2-10 días, el clopidogrel entre 5-7 días, prasugrel 7 días y el ticagrelor 3 días, para reducir la hemorragia perioperatoria (27).

En cuanto a los fármacos anticoagulantes durante muchos años los que han estado disponibles son los antivitamina $\mathrm{K}$ como la warfarina, los cuales presentan numerosas limitaciones, y requieren controles y ajustes de dosis para su rango terapéutico. Los nuevos anticoagulantes orales (NACO) son prometedores para alcanzar los rangos terapéuticos sin necesidad de monitorización y con dosis fijas que permitan reemplazar a los antivitamina $\mathrm{K}$ en el tratamiento antitrombótico. Dentro de este grupo de medicamentos están los inhibidores de la trombina como el dabigatrán, inhibidores del factor Xa como el rivaroxabán, el cual ha sido estudiado su efecto en el tratamiento de la enfermedad tromboembólica venosa, prevención de ictus isquémico en pacientes con fibrilación ventricular y en la prevención de recurrencia isquémica en pacientes con síndromes coronarios agudos (27). Se sugiere suspender estos medicamentos al menos 2 días previos a la cirugía (28).

\section{Intervenciones intraoperatorias}

\section{Medicamentos}

El utilizar medicamentos que disminuyen la fibrinólisis y protegen la membrana plaquetaria, permite reducir el sangrado postoperatorio, y el número de transfusiones de sangre durante la cirugía cardiaca.

El ácido tranexámico es uno de ellos y es aproximadamente diez veces más potente que el ácido aminocaproico, tiene una vida media más prolongada, y se une más firmemente a la molécula del plasminógeno que el ácido aminocaproico, esta característica permite su uso a dosis menores, limitando la aparición de efectos indeseables (26).

Hemodilución normovolémica aguda

Consiste en la extracción de sangre total y la sustitución de la misma con soluciones cristaloides o coloides, antes de la CEC. La sangre se extrae del paciente por una vía venosa, y se almacena en bolsas con anticoagulante (citrato ACD) para re infundirla después de la cirugía. Se ha demostrado que esta técnica, ayuda a disminuir el sangrado postoperatorio y el requerimiento de sangre homóloga. Una o dos unidades representan del 8 al 15\% del volumen circulante; una vez re infundidas, los valores de plaquetas y factores de la coagulación se incrementan en cantidad similar al valor basal. Se pueden mantener a temperatura ambiente del quirófano, teniendo precaución de no almacenarlas en el refrigerador, porque las plaquetas pierden su función (26).

Recuperación de sangre mediante rescate mecánico de eritrocitos (Cell-Saver)

Dispositivo electrónico con el que se permite recuperar hematíes con hematocritos mayores a $60 \%$, utilizado principalmenteen pacientetestigos de Jehová, quienes no autorizan transfusiones de sangre y todo aquel procedimiento en el que se prevé de un sangrado importante (26).

\section{Circuitos extracorpóreos pequeños}

El objetivo de esta intervención es reducir la hemodilución; está indicada especialmente en pacientes con alto riesgo de efectos adversos de la hemodilución como pacientes pediátricos, y adultos pequeños (29).

Se ha demostrado que los circuitos extracorpóreos más cortos, favorecen la disminución a la exposición de la sangre con superficies extrañas y el requerimiento de transfusiones sanguíneas (29).

\section{Priming retrógrado}

El uso de la sangre del paciente para cebar el circuito de CEC ha demostrado ser útil para reducir la hemodilución y las necesidades de transfusión, asimismo, mantiene niveles más 
altos de hemoglobina y no requiere medidas ni productos extras, por lo que resulta una técnica de cebado muy rentable desde un punto de vista económico. En principio, consiste en extraer al inicio del procedimiento, el máximo volumen posible de líquido de cebado del circuito y su sustitución por la propia sangre del enfermo a través de la cánula arterial de forma retrógrada. Dicho procedimiento permite eliminar o minimizar la necesidad de transfusiones de sangre durante la cirugía, aunque no podemos olvidar los riesgos que este procedimiento conlleva, como son: hipovolemia, hematocrito excesivamente elevado para procedimientos con hipotermia, hemólisis (al aumentar el hematocrito en $(E C)$, uso de vasoconstrictores y compromiso de la perfusión tisular (26).

\section{Hemoadsorción}

La denominada "tormenta citoquínica" da lugar a una cascada de fenómenos fisiopatológicos deletéreos para el paciente como son la liberación masiva de óxido nítrico que ocasiona vasodilatación periférica, además de una respuesta inotrópica negativa, pérdida de volumen intravascular, edema tisular y pulmonar, apoptosis inducida por citoquinas, consumo de plaquetas, embolización de microtrombos y fenómenos de sangrado, todo esto lleva a un fallo multiorgánico, y finalmente el fallecimiento del paciente (30).

Para ello, actualmente se ha diseñado un sistema de adsorción de mediadores proinflamatorios de la circulación (entre 10-60 kDa), disminuyendo así la respuesta inflamatoria. Estos dispositivos contienen una gran superficie de adsorción (superior a $>40.000 \mathrm{~m} 2$ o 7 estadios de fútbol), y están constituidos por esferas de un polímero poroso biocompatible capaz de eliminar citoquinas del torrente sanguíneo (TNF, IL1, IL-6 e IL-10 fundamentalmente), así como otros elementos que puedan contribuir a la respuesta inflamatoria (mioglobina, bilirrubina, exotoxinas bacterianas, mediadores activos del complemento) logrando así disminuir la intensidad de la respuesta inflamatoria y de forma secundaria la vasodilatación periférica generalizada, acidosis metabólica, riesgo de insuficiencia renal, lesión pulmonar aguda y por ende la mortalidad en áreas de cuidado crítico (30).

\section{CONCLUSIONES}

La CEC y el fenómeno de I/R contribuyen de manera significativa a la aparición de acidosis metabólica e incremento de los niveles de lactato, así como también de RLO. Estas sustancias pro inflamatorias liberadas tanto en I/R como en CEC, son determinantes para el daño miocárdico y disfunción ventricular. El daño celular provocado por la isquemia seguida de una reperfusión, conlleva a la apertura del PTPm, y como consecuencia apoptosis y necrosis de la célula.

Se puede concluir que, la cirugía cardiaca y la CEC son escenarios precisos para desencadenar una respuesta inflamatoria. Las estrategias para lograr minimizar el daño por I/R, han sido efectivas al demostrar en diferentes estudios sus resultados en la disminución de dicho fenómeno. Pero, a pesar de los esfuerzos por mejorar cada día en cuanto a la tecnología, procedimientos, técnicas quirúrgicas, y minimizar la morbilidad en el postoperatorio, aún no se ha logrado erradicar por completo el proceso inflamatorio ocasionado tanto por la cirugía cardiaca como por la CEC.

\section{REFERENCIAS BIBLIOGRÁFICAS}

1. Hall JE. Resistencia del Organismo a la Infección. En: Guyton \& Hall Tratado de fisiología médica. 12.a ed. Elsevier; 2011. p. 423-32.

2. Gil Gómez R. Estrés oxidativo y respuesta inflamatoria sistémica tras isquemia-reperfusión en el postoperatorio de cirugía cardiovascular pediátrica. Universidad de Málaga; 2014.

3. De Hert S, Moerman A. Myocardial injury and protection related to cardiopulmonary bypass. Best Pract Res Clin Anaesthesiol. 2015;29(2):13749. DOI:10.1016/j.bpa.2015.03.002

4. Javier PM. El poro de transición de permeabilidad mitocondrial (PTPm) como blanco de estrategias cardioprotector as en daño por isquemiareperfusión miocárdica: rol de los anestésicos inhalatorios. Rev Chil Anest. 2012;41(2):128-34.

5. Marín-García J, Goldenthal MJ. La mitocondria y el corazón. Rev Esp Cardiol. 2002;55(12):1293-310. DOI:10.1016/s0300-8932(02)76802-4

6. Nieves C. Terapia con Bicarbonato de Sodio en Acidosis Metabólica Desencadenada por Circulación Extracorpórea. En Bomba. 2017;1(1):817.

7. Halestrap AP. What is the mitochondrial permeability transition pore? J Mol Cell Cardiol. 2009;46(6):821-31. DOI:10.1016/j. yjmcc.2009.02.021

8. Sánchez R, Arboleda G. Mitocondria y muerte celular. NOVA. 2008;6(10). DOI:10.22490/24629448.409

9. Férez Santander SM, Márquez MF, Peña Duque MA, Ocaranza Sánchez R, de la Peña Almaguer E, Eid Lidt G. Daño miocárdico por reperfusión. Rev Esp Cardiol. 2004;57(Supl 1):9-21. DOI:10.1157/13067415 
10. Delgado-Roche L, Martínez-Sánchez G. Papel de las Especies Reactivas del Oxígeno en el Daño al Miocardio Inducido por Isquemia/Reperfusión. Rev Argent Cardiol. 2010;78(1):54-60.

11. Avello M, Suwalsky M. Radicales libres, antioxidantes naturales y mecanismos de protección. Atenea (Concepc). 2006;494:161-72. DOI:10.4067/S0718-04622006000200010

12. Mosca S. Pre y postacondicionamiento isquémicos: papel del poro de permeabilidad transitoria de la mitocondria. Rev Fed Arg Cardiol. 2011;40(2):115-20.

13. Vásquez Alarcón B. Aplicación de distintas técnicas de protección miocárdica. Rev Española Perfus. 2013;5:5-10.

14. Morita K. Surgical reoxygenation injury of the myocardium in cyanotic patients: clinical relevance and therapeutic strategies by normoxic management during cardiopulmonary bypass. Gen Thorac Cardiovasc Surg. 2012;60(9):549-56. DOI:10.1007/s11748-012-0115-2

15. Elejalde Guerra Jl. Estrés oxidativo, enfermedades y tratamientos antioxidantes. An Med Interna. 2001;18:50-9.

16. Zillo A, Carnuccio M. Protección Miocárdica durante Cirugía Cardiovascular. En: Iglesias R, Pensa $C$, editores. Cuidados Perioperatorios en Cirugía Cardiovascular. 1.a ed. Buenos Aires: Editorial Panamericana; 2010. p. 55-73.

17. England MD, Cavarocchi NC, O'Brien JF, Solis $E$, Pluth JR, Orszulak TA, et al. Influence of antioxidants (mannitol and allopurinol) on oxygen free radical generation during and after cardiopulmonary bypass. Circulation. 1986;74(5 Pt 2):III134-7.

18. Méndez EA, Segura Ten G, Zamora Rojas F, Acuña Delcore J, Gutiérrez Gutiérrez R, Pucci C. J, et al. Cardioplejia sanguínea: primera parte. Rev Costarric Cardiol. 2002;4(2):31-4.

19. Sirvinskas E, Kinderyte A, Trumbeckaite $S$, Lenkutis T, Raliene L, Giedraitis S, et al. Effects of sevoflurane vs. propofol on mitochondrial functional activity after ischemia-reperfusion injury and the influence on clinical parameters in patients undergoing CABG surgery with cardiopulmonary bypass. $\quad$ Perfusion. 2015;30(7):590-5. DOI:10.1177/0267659115571174

20. Valenzuela-Flores A, Valenzuela-Flores A, OrtegaRamírez J, Penagos-Paniagua M, Pérez-Campos JP. Alteraciones fisiopatológicas secundarias a circulación extracorpórea en cirugía cardíaca. Cir Ciruj. 2005;73(1):143-9.

21. Lespron Robles MDC. Respuesta inflamatoria sistémica en cirugía cardíaca pediátrica. Arch Cardiol Mex. 2006;76(Suppl. 2):92-9.

22. Santos-Cerquera R, Ariza Cadena F. Estrategias de protección pulmonar en cirugía cardiovascular. Rev CES Med. 2012;26(1):85-98.
23. Zillo AC. Respuesta inflamatoria difusa poscirculación extracorpórea. Rev Argent Cardiol. 1997;65(4):385-93.

24. Santos Fonseca $P L$, Pereira A, Franco $P$, Figueira I, Furtado D, Cláudio V, et al. Estrategias de ultrafiltración en CEC y su impacto en la morbilidad postoperatoria. Rev Española Perfus. 2017;63:37-48.

25. Matute $P$, Ayats MC. Valoración preoperatoria y establecimiento del riesgo clínico. En: Asociación Española de Perfusionistas (AEP), Gomar C, Mata MT, Pomar JL, editores. Fisiopatología y técnicas de circulación extracorpórea. 2.a ed. Madrid: Ergon; 2012. p. 161-271.

26. Jurado Morata A, Casado Sánchez CJ, Elías Fuentes A, Pérez López A. Estrategias para minimizar el consumo de sangre en cirugía cardiaca. Rev Española Perfus. 2015;59:39-47.

27. Páramo JA, Rábago G. Nuevos antitrombóticos. ¿Qué debe conocer el cirujano cardiovascular? Cirugía Cardiovasc. 2011;18(1):15-9. DOI: 10.1016/ S1134-0096(11)70071-7

28. Erdoes G, Martinez Lopez De Arroyabe $B$, Bolliger $D$, Ahmed AB, Koster A, Agarwal S, et al. International consensus statement on the peri-operative management of direct oral anticoagulants in cardiac surgery. Anaesthesia. 2018;73(12):1535-45. DOI: 10.1111/anae.14425

29. Arellano GA. Intervenciones para conservar la sangre en cirugía cardíaca con circulación extracorpórea. Rev Mex Enfermería Cardiológica. 2012;20(1):17-20.

30. Sánchez Ceña J, Carballo Rodríguez B, Alonso Miñambres Á, Castrillo Bustamante C, Burgos Palacios V, Sarralde Aguayo A. Novedades en cardiología crítica y cirugía cardíaca: utilización de filtros de adsorción extracorpórea de citoquinas (Citosorb®). Cirugía Cardiovasc. 2019;26(6):293-5. DOI: 10.1016/j.circv.2019.06.004

\section{ACERCA DE LOS AUTORES}

1. Eliana Cerón López. Servicio de Cirugía Cardiovascular y Torácica, Perfusión Cardiovascular, Clínica Guayaquil, Guayaquil-Ecuador.

ORCID: 0000-0002-4186-0248 